\title{
Bladder cancer cells interact with vascular endothelial cells triggering EGFR signals to promote tumor progression
}

\author{
ZHIXIN HUANG ${ }^{1,2}$, MENGZHAO ZHANG $^{1}$, GUANQIU CHEN $^{1}$, WEIYI WANG ${ }^{1}$, PU ZHANG $^{1}$, \\ YANGYANG YUE ${ }^{1}$, ZHENFENG GUAN $^{3}$, XINYANG WANG $^{1}$ and JINHAI FAN ${ }^{1}$ \\ ${ }^{1}$ Department of Urology, The First Affiliated Hospital of Xi'an Jiaotong University, Xi'an, Shaanxi 710061; \\ ${ }^{2}$ Department of Urology, Yulin No. 2 Hospital, Yulin, Shaanxi 71900; ${ }^{3}$ Department of Urology, \\ Shaanxi Provincial People's Hospital, Xi'an, Shaanxi 710068, P.R. China
}

Received August 21, 2018; Accepted January 23, 2019

DOI: 10.3892/ijo.2019.4729

\begin{abstract}
Although important progress has been made in elucidating the role of the tumor microenvironment in the development of bladder cancer, little is currently known regarding the interactions with vascular endothelial cells (ECs) that promote cancer progression. In the present study, it is reported that epidermal growth factor receptor ligands induced by the upregulation of vascular endothelial growth factor (VEGF)-A and VEGF-C via the VEGF receptor $(R) 2 /$ nuclear factor- $\kappa B$ signaling pathway in $E C s$, may trigger EGFR signaling in bladder cancer cells and promote bladder cancer progression. Furthermore, the interaction between bladder cancer cells and ECs enhanced EC recruitment though the CXCL1/CXCL5/CXCL8-CXCR2 pathway. Western blotting was used to evaluate the presence of VEGFR, EGFR and nuclear factor- $\kappa \mathrm{B}$, and reverse transcription-quantitative polymerase chain reaction was used to evaluate the expression of VEGFR ligands and EGFR ligands. The present results indicate the mechanism by which the indirect interplay between bladder cancer cells and vascular ECs promotes cancer progression, through the VEGFR2 signaling pathway in vascular ECs and through the EGFR signaling pathway in bladder cancer cells.
\end{abstract}

\section{Introduction}

Bladder cancer is the ninth most commonly diagnosed cancer, with an estimated 382,700 new cases and 150,300 mortalities recorded worldwide in 2008 (1). Approximately 90\% of bladder cancer cases are urothelial carcinoma (UC), and $70-85 \%$ of TCC are at stages Ta, T1 and carcinoma in situ.

Correspondence to: Dr Jinhai Fan, Department of Urology, The First Affiliated Hospital of Xi'an Jiaotong University, 277 Yanta West Road, Xi'an, Shaanxi 710061, P.R. China

E-mail: jinhaif029@126.com

Key words: bladder cancer, vascular endothelial cell, tumor microenvironment, epidermal growth factor receptor, vascular endothelial growth factor receptor 2
In total, $50-70 \%$ of UC cases at these stages will relapse, although there are conservative measures such as transurethral and intravesical treatment (2). The molecular mechanisms that control the development and progression of bladder cancer remain to be elucidated.

Previous studies have demonstrated that a number of intracellular signaling molecules have key roles in the progression of bladder cancer $(3,4)$. It is well-known that the phosphoinositide 3-kinase (PI3K)/protein kinase B (AKT) signaling pathway is activated in bladder cancer (3). This pathway can be misaligned by losing phosphatase and tensin homolog, and mutations in phosphatidylinositol-4,5-bisphosphate 3-kinase catalytic subunit $\alpha$ of PI3K are correlated with fibroblast growth factor receptor 3 mutations in low-grade and early-stage bladder cancer (4-7). Nuclear factor (NF)- $\kappa \mathrm{B}$ acts as a transcription regulator mediating interleukin (IL)-5, IL-8, and IL-28A expression in muscle-invasive bladder cancer, and the hypoxic microenvironment activates the $\mathrm{NF}-\kappa \mathrm{B}$ pathway, promoting the invasion and migration of T24 cells $(8,9)$. These signaling pathways serve critical roles in bladder cancer progression; however, the mechanism of $\mathrm{AKT}$ and $\mathrm{NF}-\kappa \mathrm{B}$ activation in cancer cells by the tumor microenvironment remains to be elucidated.

Tumorigenesis depends on many signaling pathways, and many types of molecules, such as extracellular matrix components, cytokines and growth factors. Epidermal growth factor receptor (EGFR) and its ligands are overexpressed in bladder cancer, and are correlated with tumor grade and stage (10). As a feature of the tumor microenvironment, tumor angiogenesis has an important role in cancer progression, and these are complex processes in which novel angiogenesis occurs in response to interactions between cancer cells and endothelial cells (ECs), and is mediated by cytokines and growth factors (11). Angiogenesis-related cytokines and growth factors secreted by cancer cells or stromal cells can directly bind to their receptors on vascular ECs and stimulate angiogenesis by promoting endothelial sprouting, differentiation and survival $(11,12)$. It has recently been demonstrated that cancer cells and vascular ECs secrete growth factors that enhance the proliferation and migration of both cells by mediating interactions between them (13-15). As such, vascular endothelial growth factor (VEGF) secreted 
by tumor or stromal cells specifically binds to its receptors, VEGF receptor(R)-1 and VEGFR-2, on EC; the PI3K/AKT and extracellular signal-regulated kinase (ERK) signaling pathways were reported to be induced by the binding of VEGF and its receptor $(16,17)$. However, the mechanisms of these interactions in bladder cancer tissue remain unclear.

Previous studies have demonstrated that cytokines secreted by vascular endothelial or cancer cells can induce proliferation, migration and invasion $(13,14)$. In the present study, it was hypothesized that cytokines or growth factors secreted by vascular ECs or cancer cells into the tumor microenvironment can promote bladder cancer cell proliferation, migration and invasion. The present study demonstrated that the VEGFR-2 signaling pathway was activated in EC-secreted EGFR ligands via the interaction between bladder cancer cells and ECs; furthermore, vascular EC-secreted EGFR ligands binding to their receptors on bladder cancer cells induced proliferation, migration and invasion through EGFR signaling and induced the secretion of CXC chemokines from bladder cancer cells, to enhance EC recruitment.

\section{Materials and methods}

Cell culture and co-culture. T24 and 253J human bladder cancer cells and human umbilical vein ECs (HUVECs) were obtained from the American Type Culture Collection (Manassas, VA, USA) and cultured in Dulbecco's modified Eagle's medium (DMEM) supplemented with $10 \%$ fetal bovine serum (FBS) (both from Invitrogen; Thermo Fisher Scientific, Inc., Waltham, MA, USA) in an atmosphere with $5 \% \mathrm{CO}_{2}$ at $37^{\circ} \mathrm{C}$.

In order to mimic the interaction between a cancer cell and $\mathrm{EC}$ in the tumor microenvironment, $0.4-\mu \mathrm{m}$ pore diameter chambers of a 6-well plate (EMD Millipore, Billerica, MA, USA) were used; 50,000 T24/253J cells were added to the lower chambers of the 6-well plate, and 50,000 HUVECs were added to the upper chambers, and cultured in DMEM (Invitrogen; Thermo Fisher Scientific, Inc.) supplemented with 10\% FBS (Invitrogen; Thermo Fisher Scientific, Inc.) in an atmosphere with $5 \% \mathrm{CO}_{2}$ at $37^{\circ} \mathrm{C}$ for $72 \mathrm{~h}$. The EGFR inhibitor lapatinib, the VEGFR inhibitor ZM $323881 \mathrm{HCl}$ and the CXCR2 inhibitor SB225002 were obtained from Selleck Chemicals (Houston, TX, USA); the NF- $\mathrm{NB}$ inhibitor PDTC was obtained from Sigma-Aldrich; Merck KGaA (Darmstadt, Germany), dissolved in dimethyl sulfoxide (DMSO) and stored at $-20^{\circ} \mathrm{C}$.

Western blotting. Following $72 \mathrm{~h}$ of co-culture, bladder cancer cell and vascular EC protein lysates were isolated with radioimmunoprecipitation assay buffer [50 mM Tris $(\mathrm{pH} 8.0)$, $150 \mathrm{mM} \mathrm{NaCl}, 0.1 \%$ SDS, $1 \% \mathrm{NP} 40$ and $0.5 \%$ sodium deoxycholate containing proteinase inhibitor $1 \%$ cocktail and $1 \mathrm{mM}$ phenylmethane sulfonyl fluoride, both from Sigma-Aldrich; Merck KGaA]. The protein concentration was calculated using a NanoDrop 1000 Spectrophotometer (Thermo Fisher Scientific, Inc.). Protein lysates were separated via $10 \%$ SDS-PAGE. Membranes were blocked with $5 \%$ non-fat milk in $1 \mathrm{X}$ TBS containing $0.3 \%$ Tween-20 (TBST), incubated with primary antibodies (Table I) overnight at $4^{\circ} \mathrm{C}$ and washed with 1X TBST (pH 7.6). Membranes incubated with IRDye ${ }^{\circledR}$-conjugated goat anti-rabbit (cat. no. 926-32211) or goat anti-mouse secondary antibodies (cat. no. 926-68070; both LI-COR Biosciences, Lincoln, NE, USA) diluted at 1:1,000 in 5\% skimmed milk were then applied for $1 \mathrm{~h}$ at room temperature, followed by washing as described previously, in a dark room, drying with neutral absorbent paper and scanning with an Odyssey detection system (LI-COR Biosciences). GAPDH was used as the loading control.

Following $48 \mathrm{~h}$ of co-culture, the medium was extracted and supplemented with FBS-free DMEM, and the upper chambers were removed and placed into a 6 -well plate. The EGFR inhibitor lapatinib (10 $\mu \mathrm{M}$ in medium) or $\mathrm{NF}-\kappa \mathrm{B}$ inhibitor PDTC (5 $\mu \mathrm{M}$ in medium) were added to the lower chambers, and the VEGFR inhibitor ZM $323881 \mathrm{HCl}$ (10 $\mathrm{nM}$ in medium) or the inhibitor CXCR2 SB225002 (2 $\mu \mathrm{M}$ in medium) were added to the upper chambers for a further $24 \mathrm{~h}$ at $37^{\circ} \mathrm{C}$ prior to protein or total mRNA extraction.

Reverse transcription-quantitative polymerase chain reaction $(R T-q P C R)$. Total RNA of the cells was isolated using RNAfast 200 reagent (Shanghai Fastagen Biotechnology Co., Ltd., Shanghai, China) and quantitated by measuring the absorbance at a wavelength of $260 \mathrm{~nm}$. The RNA $(2 \mu \mathrm{g})$ sample was reverse transcribed with 5X PrimeScript RT Master Mix (2 $\mu$ l; Takara Biotechnology Co., Ltd., Dalian, China), RNase-free $\mathrm{dH}_{2} \mathrm{O}(7 \mu \mathrm{l})$ and total RNA $(1 \mu \mathrm{l})$ were mixed and reacted at $37^{\circ} \mathrm{C}$ for $16 \mathrm{~min}$ and $85^{\circ} \mathrm{C}$ for $5 \mathrm{sec}$. qPCR was then performed using the SYBR Premix Ex Taq ${ }^{\mathrm{TM}}$ II system (Takara Biotechnology Co., Ltd.) and the Bio-Rad CFX96 ${ }^{\mathrm{TM}}$ Real-time system (Bio-Rad Laboratories, Inc.). SYBR Premix Ex Taq II $(12.5 \mu \mathrm{l}), 1 \mu \mathrm{l}$ sense primer $(10 \mu \mathrm{M}), 1 \mu \mathrm{l}$ anti-sense primer (10 $\mu \mathrm{M}) 2 \mu \mathrm{l}$ cDNA solution and $8.5 \mu \mathrm{l}$ RNase-free water were mixed together. The following thermocycling protocol was used with three stages, including pre-degeneration for $95^{\circ} \mathrm{C}$ for $30 \mathrm{sec}$, one repeat; PCR amplification at $95^{\circ} \mathrm{C}$ for $5 \mathrm{sec}$ followed by $60^{\circ} \mathrm{C}$ for $30 \mathrm{sec}, 40$ repeats; and dissociation at $95^{\circ} \mathrm{C}$ for $15 \mathrm{sec}$ followed by $60^{\circ} \mathrm{C}$ for $30 \mathrm{sec}$ and $95^{\circ} \mathrm{C}$ for $15 \mathrm{sec}$. GAPDH was used as the loading control to balance the quantity of the samples, and the gene expression was normalized to the GAPDH to calculate relative expression level using the $2^{-\Delta \Delta \mathrm{Cq}}$ method (18). The gene-specific primers used are listed in Table II.

Colony formation assay. Chambers (0.4- $\mu \mathrm{m}$ pore diameter) were obtained from EMD Millipore. A total of 1,000 T24/253J cells were added to the lower chambers of the 6-well plate, and 1,000 HUVECs were added to the upper chambers, then cultured in DMEM supplemented with $10 \%$ FBS in an atmosphere with $5 \% \mathrm{CO}_{2}$ at $37^{\circ} \mathrm{C}$. In the control group, 1,000 $\mathrm{T} 24 / 253 \mathrm{~J}$ cells were added to the lower chambers of the 6 -well plate, and the upper chambers were not seeded with HUVECs. Following 14 days, the plates were washed with PBS, fixed in $4 \%$ formalin, stained with crystal violet solution for $15 \mathrm{~min}$ at room temperature, and washed with PBS to remove the excess dye. Cells counted from five randomly selected fields were counted via light microscopy at x100 magnification.

MTT assay. Briefly, a 24-well Transwell plate was used (0.4- $\mu \mathrm{m}$ pore diameter; EMD Millipore, Schaffhausen, Switzerland) for MTT assay. Following $48 \mathrm{~h}$ of co-culture, the 
Table I. Primary antibodies used for western blotting.

\begin{tabular}{|c|c|c|}
\hline Primary antibodies (dilution) & Catalogue number & Supplier \\
\hline Anti-EGFR & D38B1 & Cell Signaling Technology, Inc., Danvers, MA, USA \\
\hline Anti-ErbB2 & D8F12 & Cell Signaling Technology, Inc., Danvers, MA, USA \\
\hline Anti-ErbB3 & D22C5 & Cell Signaling Technology, Inc., Danvers, MA, USA \\
\hline Anti-ErbB4 & 111B2 & Cell Signaling Technology, Inc., Danvers, MA, USA \\
\hline Anti-p-EGFR (Tyr1068) & D7A5 & Cell Signaling Technology, Inc., Danvers, MA, USA \\
\hline Anti-p-ErbB2 (Tyr1221/1222) & $6 \mathrm{~B} 12$ & Cell Signaling Technology, Inc., Danvers, MA, USA \\
\hline Anti-p-ErbB3 (Tyr1289) & D1B5 & Cell Signaling Technology, Inc., Danvers, MA, USA \\
\hline Anti-p-ErbB4 (Tyr1284) & 21A9 & Cell Signaling Technology, Inc., Danvers, MA, USA \\
\hline Anti-AKT (pan) & $11 \mathrm{E} 7$ & Cell Signaling Technology, Inc., Danvers, MA, USA \\
\hline Anti-p-AKT (Ser473) & D9E & Cell Signaling Technology, Inc., Danvers, MA, USA \\
\hline Anti- ERK1/2 & 137F5 & Cell Signaling Technology, Inc., Danvers, MA, USA \\
\hline Anti-p-ERK1/2 (Thr202/Tyr204) & D13.14.4E & Cell Signaling Technology, Inc., Danvers, MA, USA \\
\hline Anti-STAT3 & $\mathrm{D} 3 \mathrm{Z} 2 \mathrm{G}$ & Cell Signaling Technology, Inc., Danvers, MA, USA \\
\hline Anti-p-STAT3 (Tyr705) & D3A7 & Cell Signaling Technology, Inc., Danvers, MA, USA \\
\hline Anti-NF- $x$ B p 65 & D14E12 & Cell Signaling Technology, Inc., Danvers, MA, USA \\
\hline Anti-p-NF- $x B$ p65 & $93 \mathrm{H} 1$ & Cell Signaling Technology, Inc., Danvers, MA, USA \\
\hline Anti-MMP-2 & D2O4T & Cell Signaling Technology, Inc., Danvers, MA, USA \\
\hline Anti-MMP-9 & $\mathrm{D} 6 \mathrm{O} 3 \mathrm{H}$ & Cell Signaling Technology, Inc., Danvers, MA, USA \\
\hline Anti-N-cadherin & D4R1H & Cell Signaling Technology, Inc., Danvers, MA, USA \\
\hline Anti-ZEB 1 & D80D3 & Cell Signaling Technology, Inc., Danvers, MA, USA \\
\hline Anti-Survivin & 71G4B7 & Cell Signaling Technology, Inc., Danvers, MA, USA \\
\hline Anti-GAPDH & $\mathrm{KC}-5 \mathrm{G} 4$ & Kangchen BioTech Co., Ltd., Shanghai, China \\
\hline Anti-CXCR2 & ab14935 & Abcam, Cambridge, UK \\
\hline Anti-p-VEGFR2 (Tyr1059) & D5A6 & Cell Signaling Technology, Inc., Danvers, MA, USA \\
\hline Anti-p-VEGFR2 (Tyr1175) & D5B11 & Cell Signaling Technology, Inc., Danvers, MA, USA \\
\hline Anti-p-VEGFR2 (Tyr996) & 2474 & Cell Signaling Technology, Inc., Danvers, MA, USA \\
\hline Anti-VEGFR2 & D5B1 & Cell Signaling Technology, Inc., Danvers, MA, USA \\
\hline
\end{tabular}

All primary antibodies were derived from rabbits and used at a dilution of 1:1,000. EGFR, epidermal growth factor receptor; p, phosphorylated; AKT, protein kinase B; ERK, extracellular signal-regulated kinase; STAT3, signal transducer and activator of transcription factor 3; NF, nuclear factor; MMP, matrix metalloprotein; ZEB, zinc finger E-box-binding homeobox; VEGFR, vascular endothelial growth factor receptor.

upper chambers were removed, $100 \mu 15 \mathrm{mg} / \mathrm{ml}$ MTT solution was added to each well, and the plate was then incubated at $37^{\circ} \mathrm{C}$ for a further $4 \mathrm{~h}$. Thereafter, the medium was aspirated and 1,000 $\mu 1$ DMSO was added to each well. The microtitre plate was placed on a shaker in order to dissolve the dye. After the formazan crystals had dissolved, the absorbance was determined spectrophotometrically at $490 \mathrm{~nm}$ on an ELX800 UV universal microplate reader (BioTek Instruments, Inc., Winooski, VT, USA).

Migration and invasion assay. Cell migration and invasion was assessed using a Transwell Boyden chamber assay. The chambers $(8-\mu \mathrm{m}$ pore diameter) were obtained from EMD Millipore. For migration assay, following co-culture $48 \mathrm{~h}$ later, $400 \mu$ l FBS-free DMEM suspension with 10,000 T24 cells and 50,000 253J cells were added to the upper chamber in the 24-well plate, and $800 \mu \mathrm{l}$ DMEM without FBS was added to the lower chambers. Following $24 \mathrm{~h}$ incubation at $5 \% \mathrm{CO}_{2}$ and $37^{\circ} \mathrm{C}$, cells were fixed with $4 \%$ formalin for $30 \mathrm{~min}$ at $37^{\circ} \mathrm{C}$, stained with crystal violet $(0.01 \%$ in ethanol) for $10 \mathrm{~min}$ at room temperature, washed three times and counted under an inverted light microscope. Five random sections imaged for each well at x200 magnification and the average number of cells was calculated. For analysis of invasion, suspension in the upper chambers contained $60 \mu 1$ mixture [FBS-free DMEM:Matrigel (Sigma-Aldrich; Merck KGaA) = 12:1], and either 10,000 T24 or 5,000 253J cells, the incubation time was $36 \mathrm{~h}$, and all other steps were the same as for the migration analysis.

HUVEC recruitment. In order to monitor the HUVEC recruitment of cancer cell lines, Boyden chambers were used (8- $\mu \mathrm{m}$ pore diameter; EMD Millipore). A total of 10,000 T24 cells and 50,000 253J cells, which educated by co-culture or control for $36 \mathrm{~h}$, was seeded into the 24-well plate until its adhesion to the bottom, followed by planting 10,000 HUVECs into the upper chambers. Following incubation for $24 \mathrm{~h}$ at $37^{\circ} \mathrm{C}$, the number of HUVECs counted as the Boyden chamber assay.

Statistical analysis. All statistical analyses were performed using GraphPad Prism version 5.0 software (GraphPad Software, Inc., La Jolla, CA, USA). All data were reported 
Table II. Primers used for reverse transcription-quantitative polymerase chain reaction.

\begin{tabular}{llcl}
\hline $\begin{array}{l}\text { Gene } \\
\text { ID }\end{array}$ & Gene & Primers & \multicolumn{1}{c}{ Sequence (5'-3') } \\
\hline 2597 & GAPDH & F & GGAGCGAGATCCCTCCAAAAT \\
& & R & GGCTGTTGTCATACTTCTCATGG \\
1950 & EGF & F & TCCTCACCCGATAATGGTGGA \\
& & R & CCAGGAAAGCAATCACATTCC \\
7039 & TNFA & F & AGATAGACAGCAGCCAACCCTGA \\
& & R & CTAGGGCATTCTGCCCATC \\
1839 & HBEGF & F & ATCGTGGGGCTTCTCATGTTT \\
& & R & TTAGTCATGCCCAACTTCACTTT \\
685 & BTC & F & CTAGGTGCCCCAAGCAATACA \\
& & R & GCAGACACCGATGACCAAAATA \\
374 & AREG & F & GTGGTGCTGTCGCTCTTGATA \\
& & R & CCCCAGAAAATGGTTCACGCT \\
2069 & EREG & F & GGACAGTGCATCTATCTGGTGG \\
& & R & TTGGTGGACGGTAAAAAGAAGT \\
255324 & EPGN & F & ATGGCTTTGGGAGTTCCAATATC \\
& & R & TCCTTCTATGTTGTCAGCTTGC \\
7422 & VEGF-A & F & AGGGCAGAATCATCACGAAGT \\
& & R & AGGGTCTCGATTGGATGGCA \\
7424 & VEGF-C & F & GAGGAGCAGTTACGGTCTGTG \\
& & R & TCCTTTCCTTAGCTGACACTTGT \\
2919 & CXCL1 & F & AACCGAAGTATAGCCACAC \\
& & R & GTTGGATTTGTCACTGTTCAGC \\
6374 & CXCL5 & F & AGCTGCGTTGCGTTTGTTTAC \\
& & R & TGGCGAACACTTGCAGATTAC \\
3576 & CXCL8 & F & ACTGAGAGTGATTGAGAGTGGAC \\
& & R & AACCCTCTGCACCCAGTTTTC \\
\hline
\end{tabular}

F, forward; R, reverse; EGF, epidermal growth factor; HBEGF, heparinbinding EGF-like growth factor; AREG, amphiregulin; EREG, epiregulin; BTC, betacellulin; TNFA, tumor necrosis factor $\alpha$; EPGN, epithelial mitogen; VEGF, vascular endothelial growth factor.

as the mean \pm standard error, and significant differences were measured using an unpaired two-sided Student's t-test or differences in each group were analyzed by one-way analysis of variance, followed by Dunnett's t-test for separate comparisons. Kaplan-Meier analysis was used to estimate cluster of differentiation (CD)31 and EGFR protein expression in The Cancer Genome Atlas (TCGA) bladder cancer PRAD_exp_HiSeqV2 ( $\mathrm{n}=345)$ dataset (http://firebrowse.org/). Based on the mean number of CD31 protein expression, 0.023 , samples were divided into two groups and the group with $>0.023$ had the lower survival ratio. Based on the mean number of EGFR protein expression, - 0.003248 , samples were divided into two groups, and the group with $>-0.003248$ had the lower survival ratio. Spearman's correlation analysis was used to identify and correlation between EGFR pY1068 expression and AKT pS473 and NF- $\mathrm{KB}$ p65 pS536 expression in TCGA dataset. $\mathrm{P}<0.05$ was considered to indicate a statistically significant difference.

\section{Results}

Interactions between ECs and bladder cancer cells increase cell viability and malignancy. Research of bladder cancer has demonstrated that angiogenesis has an important role in bladder cancer progression (19). It was demonstrated that CD31 had a strong positive expression in bladder cancer, and a weak negative expression in normal bladder tissue (Fig. 1A). Furthermore, Kaplan-Meier analysis was used to estimate CD31 protein expression in the TCGA bladder cancer PRAD_exp_HiSeqV2 $(n=345)$ dataset, which indicated that CD31 expression is an independent risk factor for patients with bladder cancer (based on the mean number of CD31 protein expression, 0.023 , samples are divided into two groups, and the group $>0.023$ has a lower survival ratio; $\mathrm{P}<0.001$; Fig. 1B).

It has previously been demonstrated that the number of ECs are increased according to tumor progression and are negatively associated with the prognosis of bladder cancer (19). In the present study it was hypothesized that ECs serve other direct or indirect roles in bladder cancer apart from the known metabolism-associated roles (such as oxygen supply). The Boyden chamber system $(0.4-\mu \mathrm{m}$ pore diameter) was used to co-culture bladder cancer cells (T24/253J) with ECs (HUVECs) to mimic the tumor microenvironment (Fig. 1C). Colony-formation (Fig. 1D and E) and MTT assays (Fig. 1F and G) indicated that co-culture contributes to cancer cell viability. The results of the Boyden chamber assay indicated that the co-culture resulted in the enhanced malignancy of T24/253J cells (Fig. 1H and I).

Co-culture treatment activates EGFR signaling in bladder cancer cells. Overexpression of ErbB family proteins has been reported in a number of studies on bladder cancer, which indicated that there was a significant association between clinical outcome and tumor grade (20). EGFR protein expression was analyzed in clinical specimens from the human protein atlas (www.proteinatlas.org). It was demonstrated that EGFR had strong positive expression in bladder cancer and weak negative expression in normal bladder tissues (Fig. 2A). Furthermore, Kaplan-Meier analysis was used to estimate EGFR protein expression in the TCGA bladder cancer PRAD_exp_HiSeqV2 $(\mathrm{n}=345)$ dataset (http://firebrowse.org/), which indicated that EGFR expression is a risk factor for patients with bladder cancer (Fig. 2B). To obtain insight into the role of the EGFR family in bladder cancer cells, EGFR family expression in response to the co-culture of HUVEC and T24/253J cells was determined. Western blotting revealed that EGFR signaling was induced by co-culture treatment (Fig. 2C). In addition, it was demonstrated that downstream EGFR signaling was induced, including AKT, signal transducer and activator of transcription factor 3 (STAT3), ERK and NF- $\kappa$ B. It was also attempted to analyze the gene expression of matrix metalloproteinase (MMP)2, MMP9, zinc finger E-box-binding homeobox (ZEB)-1, survivin and $\mathrm{N}$-cadherin following co-culture, using western blotting (Fig. 2D). As presented in Fig. 2E, co-culture upregulated MMP2, MMP9, ZEB-1, survivin and N-cadherin gene expression. Spearman's rank correlation analysis was performed in the TCGA dataset, which revealed a significant positive correlation between the EGFR pY1068 and AKT pS473, and NF-кB p65 pS536 (Fig. 2F and G). 
A

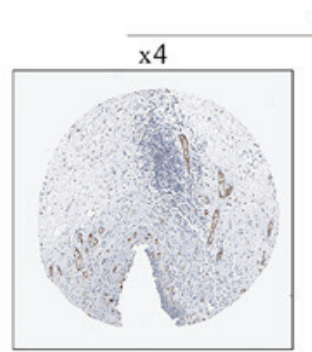

Urinary bladder normal tissue
CD31

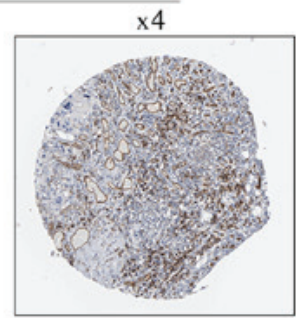

Urinary bladder cancer tissue
B

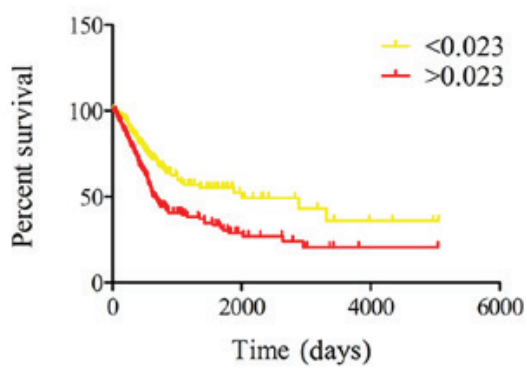

C

Model of Co-culture system

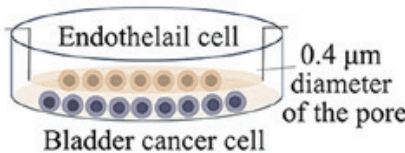

F
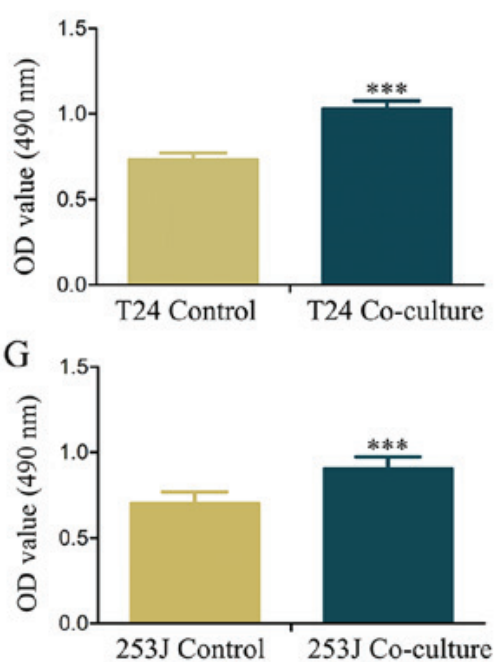

D
$\mathrm{T} 24$

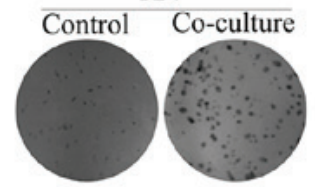

E

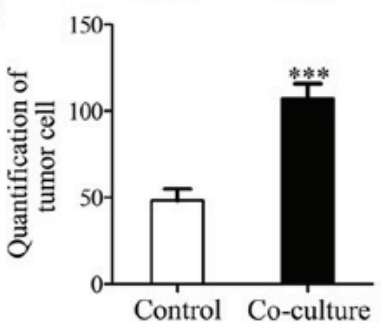

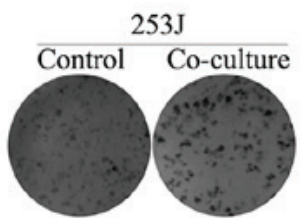

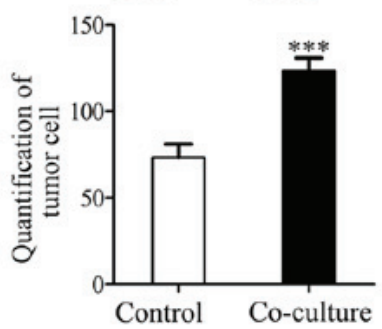

$\mathrm{H}$
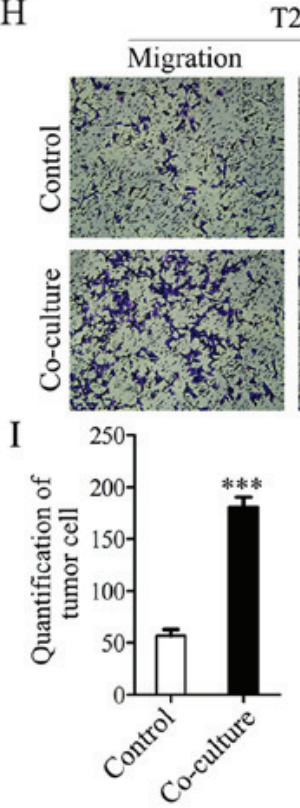

$\mathrm{T} 24$
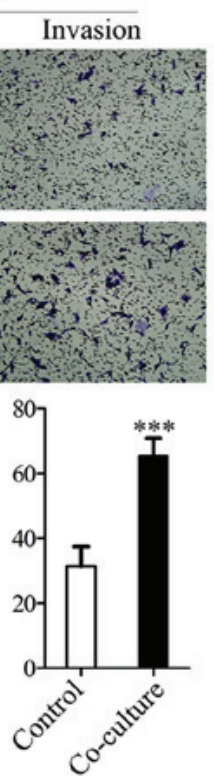

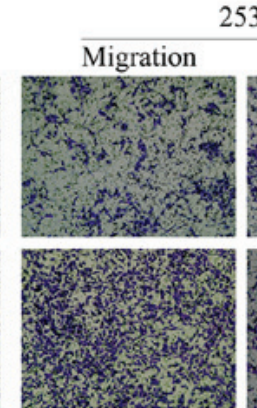

$253 \mathrm{~J}$
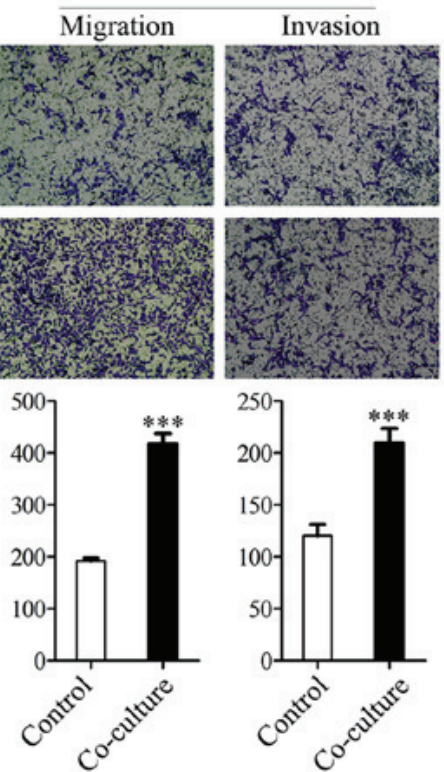

Figure 1. CD31 as a marker of EC expression is an independent risk factor for patients with bladder cancer, and co-culture of ECs with bladder cancer cells enhances cancer cell proliferation and malignancy. (A) CD31 expression in normal bladder tissue and bladder carcinoma specimens. Images were taken from the Human Protein Atlas (http://www.proteinatlas.org) online database. (B) Kaplan-Meier analysis estimates CD31 protein expression in the Cancer Genome Atlas bladder cancer PRAD_exp_HiSeqV2 (n=345) dataset (http://firebrowse.org/), and indicated that CD31 expression is an independent risk factor for patients with bladder cancer (based on the mean number of CD31 protein expression, 0.023 , samples are divided into two groups and the group $>0.023$ has the lower survival ratio; $\mathrm{P}<0.001$ ). (C) Illustration of the co-culture system. (D and E) Colony formation assay determined the effect of co-culture on cell proliferation (magnification, $\mathrm{x} 100$ ). (F and G) MTT assay determined T24/253J bladder cancer cell proliferation following co-culture. (H and I) Transwell migration assay revealed that co-culture promotes cancer cell migration and invasion (magnification, $\mathrm{x} 200$ ). ${ }^{* * * *} \mathrm{P}<0.001$ vs. control. CD, cluster of differentiation; EC, endothelial cell.

Inhibition of EGFR signaling in T24/253J cells attenuates co-culture induced malignancy and proliferation. In order to demonstrate the mechanism of EGFR signaling in the co-culture system, lapatinib, an inhibitor of EGFR and Her2, was used to inhibit this signaling pathway. Lapatinib resulted in the attenuation of EGFR signaling activated by co-culture compared with co-culture $+0.5 \%$ DMSO (Fig. 3A), which was accompanied by attenuated malignancy (Fig. 3B and C). In addition, this signaling inhibition ameliorated the co-culture induced proliferative ability of T24/253J cells (Fig. 3D-G). This indicated that the EGFR pathway was a key regulator of interactions between bladder cancer cells and ECs. 
A

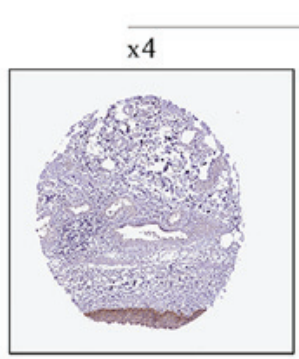

Urinary bladder normal tissue
EGFR

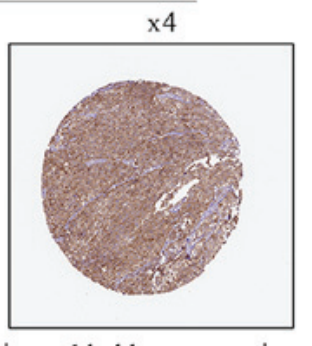

Urinary bladder cancer tissue
B

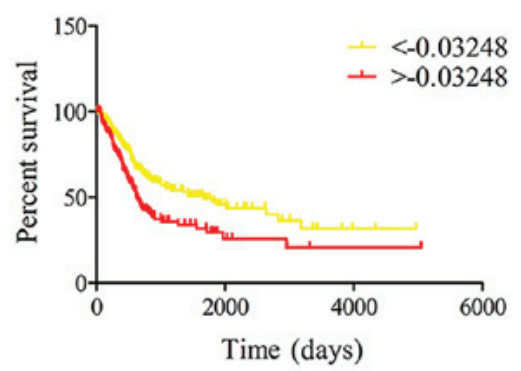

$\mathrm{E}$

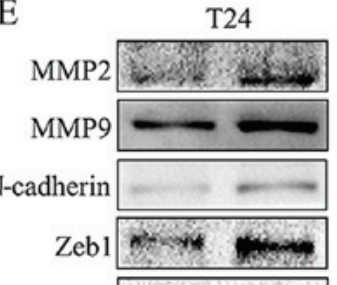

$253 \mathrm{~J}$

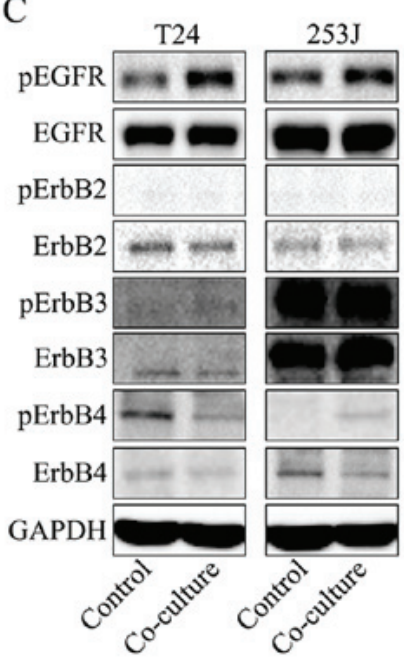

$\mathrm{D}$

$\mathrm{T} 24$

$253 \mathrm{~J}$
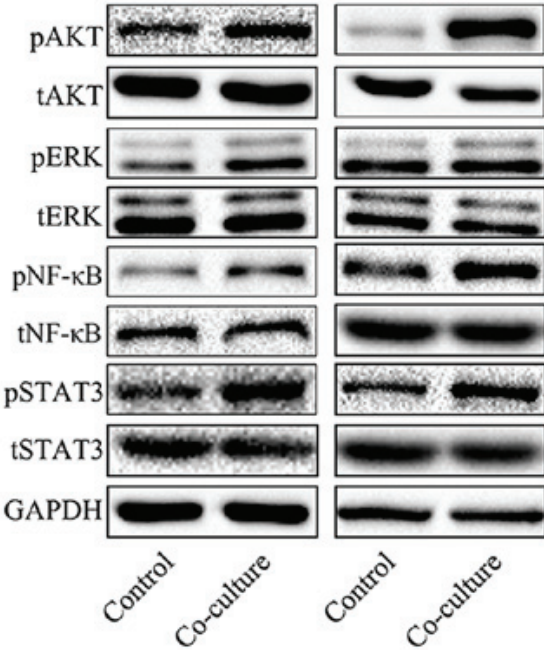

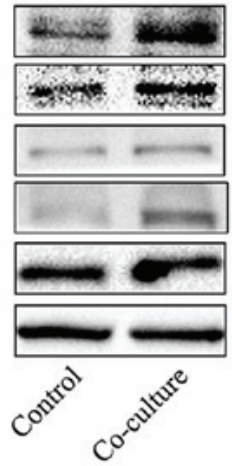

$\mathrm{F}$

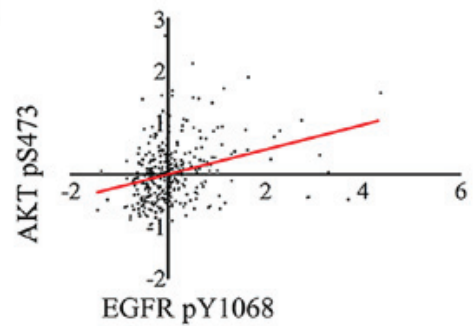

G

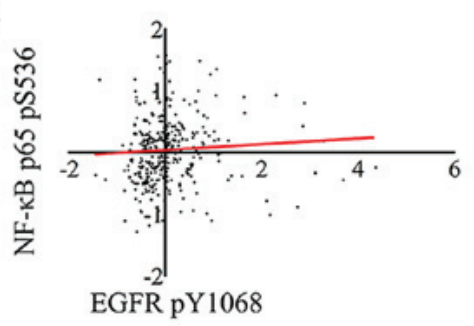

Figure 2. EGFR signaling in bladder cancer cells is triggered by EGFR ligands secreted by endothelial cells and promotes bladder cancer progression. (A) EGFR expression in normal bladder tissue and bladder carcinoma specimens. Images were taken from the Human Protein Atlas (http://www.proteinatlas.org) online database. (B) Kaplan-Meier analysis estimated EGFR protein expression in TCGA bladder cancer PRAD_exp_HiSeqV2 (n=345) dataset (http://firebrowse.org/), and indicated that EGFR expression is an independent risk factor for patients with bladder cancer (based on the mean number of EGFR protein expression -0.003248 , samples were divided into two groups, the group $>-0.003248$ has the lower survival ratio; $\left.{ }^{* * *} \mathrm{P}<0.001\right)$. (C) EGFR signaling was induced by co-culture treatment. Western blot analysis of EGFR family protein expression following co-culture treatment revealed that EGFR pY1068 was upregulated compared with the control group. (D) Downstream EGFR signaling was activated by co-culture treatment. Western blot analysis indicates that co-culture induces downstream EGFR signaling: AKT pS473, NF-kB p65 pS536, STAT3 pY705 and pERK1/2 (Thr202/Tyr204) were all upregulated. (E) Western blotting indicated that the co-culture treatment of T24/253J leads to upregulation of MMP2, MMP9, ZEB-1, survivin and N-cadherin. Correlation between EGFR pY1068 expression and (F) AKT pS473 and (G) NF-kB p65 pS536 expression in TCGA dataset. EGFR, epidermal growth factor receptor; AKT, protein kinase B; NF, nuclear factor; STAT3, signal transducer and activator of transcription factor 3; ERK, extracellular signal-regulated kinase; p, phosphorylated; MMP, matrix metalloprotein; ZEB, zinc finger E-box-binding homeobox; TCGA, The Cancer Genome Atlas; t, total.

Furthermore, the inhibition of EGFR signaling also led to the reversal of epithelial-mesenchymal transition (EMT) marker expression including the downregulation of MMP2, MMP9, $\mathrm{N}$-cadherin, ZEB-1 and survivin (Fig. 3H). These results are in accordance with those from a previous study (10), which indicated that the EGFR pathway was a key EMT regulator.

Co-culture system activates VEGFR2 signaling in EC and upregulates EGFR ligand expression. Both EGFR and its ligands are transmembrane proteins. Previous studies have provided evidence that ligand-receptor binding activates the cytoplasmic tyrosine kinase domains of EGFR, resulting in signal transduction to promote cell proliferation, migration, differentiation and survival (21). The present results indicated that the co-culture system activates EGFR signaling. In order to elucidate the mechanism underlying this phenomenon, EGFR ligands were monitored in control and co-culture systems. As indicated, the EGFR ligands except for heparin-binding EGF-like growth factor, have different expression profiles in HUVECs, when compared with in T24 or 253J cells (Fig. 4A and B). This indicated that there is a feature of the co-culture system that may contribute to the upregulation of EGFR ligands. 


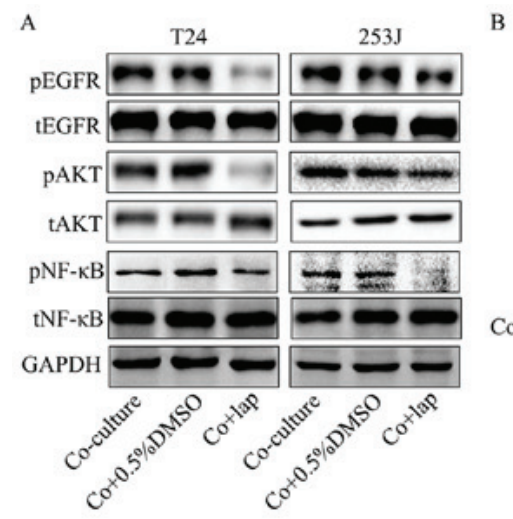

B
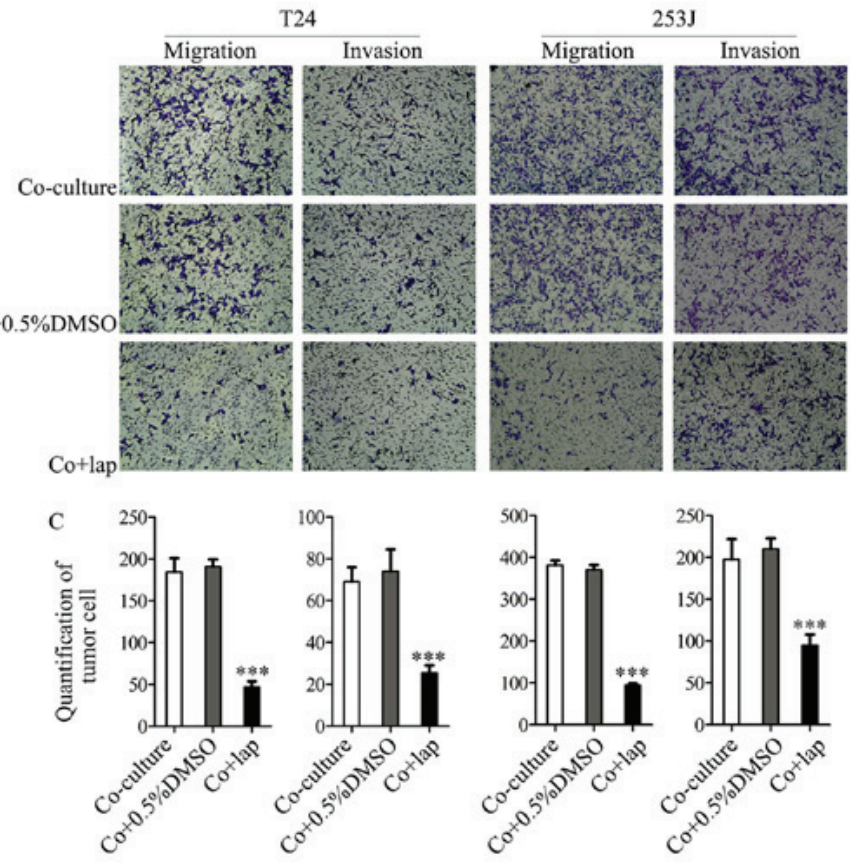

D

T24

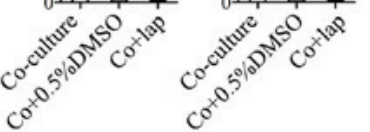

Co-culture Co+0.5\%DMSO Co+lap Co-culture Co+0.5\%DMSO Co+lap
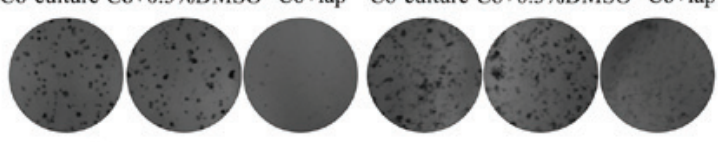

$\mathrm{E}$
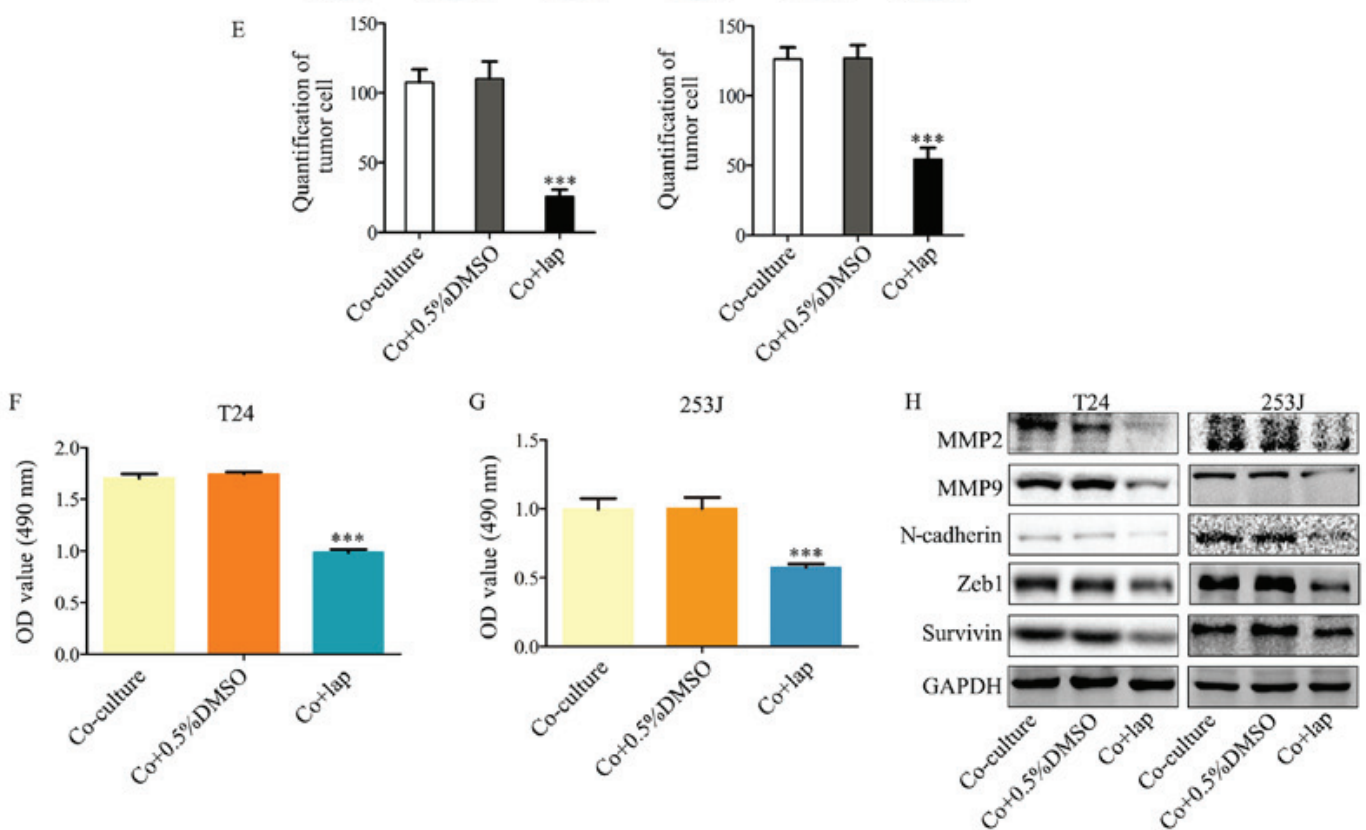

Figure 3. Inhibition of EGFR signaling in bladder cancer cells by lapatinib in a co-culture system abrogates co-culture induced cancer cell malignancy and proliferation. (A) Western blot analysis indicates that downstream EGFR signaling was inhibited by co-culture treatment with lapatinib. (B and C) Transwell migration assays indicated that co-culture induced malignancy of T24/253J is attenuated in the presence of lapatinib (magnification, x200). (D and E) Colony formation assay indicated that the co-culture induced enhanced proliferation of T24/253J is ameliorated in the absence of EGFR signaling (magnification, x200). (F and G) MTT assay determination of bladder cancer T24/253J cell proliferation following co-culture treatment with lapatinib. (H) Western blot analysis indicates that in co-culture system the proteins MMP2, MMP9, ZEB-1, survivin and N-cadherin were downregulated by inhibited EGFR signaling. ${ }^{* * * *} \mathrm{P}<0.001$ vs. co-culture and co+0.5\%DMSO. EGFR, epidermal growth factor receptor; co+0.5\%DMSO, co-culture + 0.5\% dimethyl sulfoxide; MMP, matrix metalloprotein; ZEB, zinc finger E-box-binding homeobox; co+lap, co-culture + lapatinib; OD, optical density; p, phosphorylated; t, total; AKT, protein kinase B; NF, nuclear factor.

It has previously been demonstrated that VEGFs and VEGFRs have important roles in both physiological vascular development and pathological diseases, for example, tumor angiogenesis. VEGFR-2 (Flk-1/KDR) is expressed in ECs and lymphatic ECs (22). VEGFR-2 has three ligands: VEGF-A, VEGF-C and VEGF-D. These ligands bind to VEGFR to induce activation of intracellular signaling cascades results in proliferation, migration, survival and 


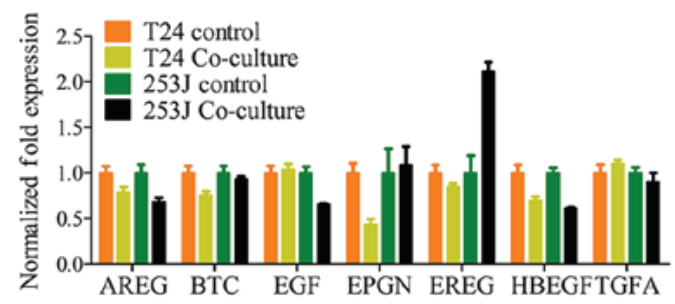

B

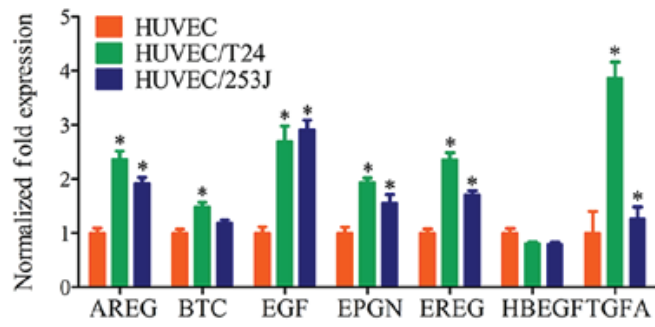

D

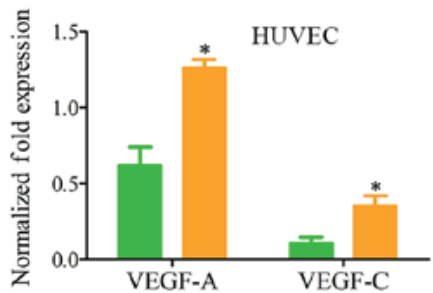

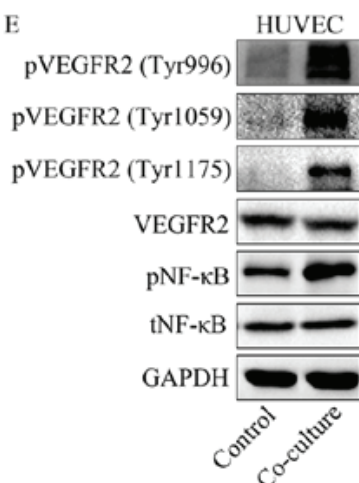

Control

Co-culture

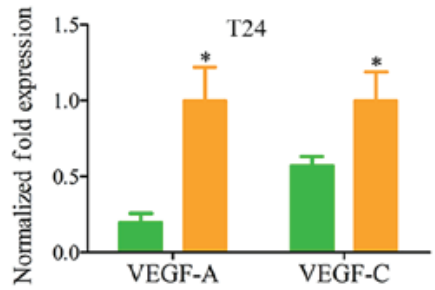

\section{F Co-culture with T24 $\mathrm{ZM} 323881 \mathrm{HCl}$ PDTC}

pVEGFR2 (Tyr1059)

pVEGFR2 (Tyr1 175)

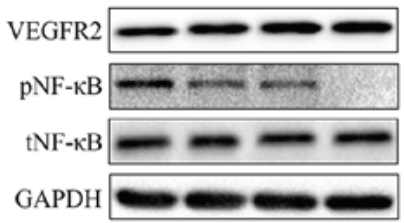

G

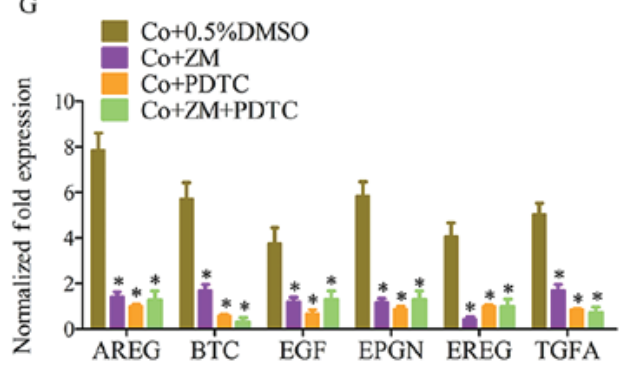

Figure 4. The VEGFR2 pathway of endothelial cells is triggered by VEGF-A and VEGF-C in co-culture system, and induces EGFR ligand upregulation. (A) RT-qPCR for screening EGFR ligands in T24/253J following co-culture with HUVECs. EREG are significantly elevated in $253 \mathrm{~J}$ by co-culture. (B) RT-qPCR for screening EGFR ligands in HUVECs following co-culture with T24/253J. Except for HBEGF, EGFR ligands are significantly elevated in HUVEC after co-culture; co-culture vs. control, "P<0.05; RT-qPCR for screening VEGF-A and VEGFR-C in (C) T24 and (D) HUVECs following co-culture; co-culture vs. control, $\mathrm{P}<0.05$. (E) Western blot analysis demonstrated that VEGFR2 signaling was induced in HUVECs by co-culture with T24. pVEGFR2-Tyr996, pVEGFR2-Tyr1059, and pVEGFR2-Tyr1175 were upregulated compared with the control group, and the downstream NF- $\kappa B$ pathway was upregulated compared with the control group. (F) Western blot analysis indicates that in the co-culture system, VEGFR2 signaling inhibition by ZM $323881 \mathrm{HCL}$ and NF- $\mathrm{kB}$ pathway was inhibited by PDTC. (G) RT-qPCR for screening the expression of EGFR ligands in HUVECs following co-culture with T24/253J, followed by inhibition of the EGFR-NF-kB pathway. "P<0.05 vs. co+0.5\%DMSO. VEGF, vascular endothelial growth factor; R, receptor; RT-qPCR, reverse transcription-quantitative polymerase chain reaction; EGFR, epidermal growth factor receptor; HUVEC, human umbilical vein endothelial cell; HBEGF, heparin-binding EGF-like growth factor; NF, nuclear factor; Co+0.5\%DMSO, co-culture + $0.5 \%$ dimethyl sulfoxide; Co+ZM, co-culture+ZM 323881 HCl; Co+PDTC, co-culture+PDTC; Co+ZM+PDTC, co-culture+ZM 323881 HCl+PDTC; p, phosphorylated; t, total; EGF, epidermal growth factor; AREG, amphiregulin; EREG, epiregulin; BTC, betacellulin; TNFA, tumor necrosis factor $\alpha$; EPGN, epithelial mitogen.

increased permeability (23). With the co-culture system it was demonstrated that both T24 cancer cells and HUVECs have a high level of VEGF-A and VEGF-C expression compared with the control group (Fig. 4C and D). In addition, VEFGR2 protein expression was analyzed in the co-culture system, which revealed that VEGFR2 signaling in HUVECs was induced by co-culture (Fig. 4E and F). A number of techniques, such as cDNA microarray analysis, have previously been used to identify genes that are upregulated in ECs following stimulation with VEGF (23). The present findings suggest that the VEGFR2 signaling pathway has an effect on the expression of those EGFR ligands. Education of HUVECs by co-culture was repeated in the presence of ZM $323881 \mathrm{HCl}$ (an inhibitor of VEGFR2) and PDTC, and RT-qPCR was used to assess the expression of EGFR ligands in tumor cells (Fig. 4G). present study it was demonstrated that T24/253J cells educated by co-culture exhibited enhanced HUVEC recruitment ability compared with the control group (Fig. 5A and B). It is known that the CXC chemokine has an important role in the processes of tumor angiogenesis and cell recruitment. Subsequently, it was observed that CXCL1, CXCL5 and CXCL8 were upregulated in cancer cells when co-cultured with HUVECs (Fig. 5C), and that CXCR2 (the receptor for CXCL1/5/8) was upregulated in HUVECs when co-cultured with cancer cells (Fig. 5D). In addition, CXCR2 was inhibited in HUVECs by co-culturing for $36 \mathrm{~h}$ with SB225002 (an inhibitor of CXCR2); the results indicated that the co-culture system enhanced the EC recruitment ability through the CXC chemokine and its receptor (Fig. 5E and F). However, the mechanisms involved in the upregulation of CXCL1, CXCL5 and CXCL8 in cancer cells are not fully understood. Our previous study has demonstrated that the EGFR signaling pathway and its downstream signaling was activated by 

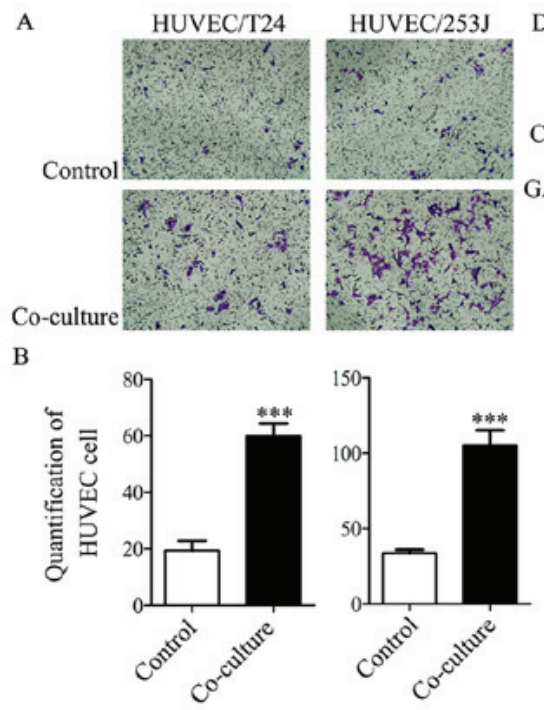

$\mathrm{C}$

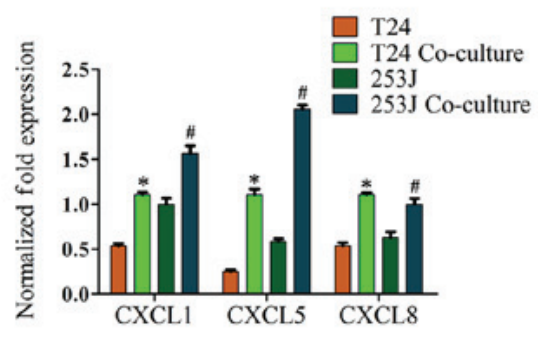

H
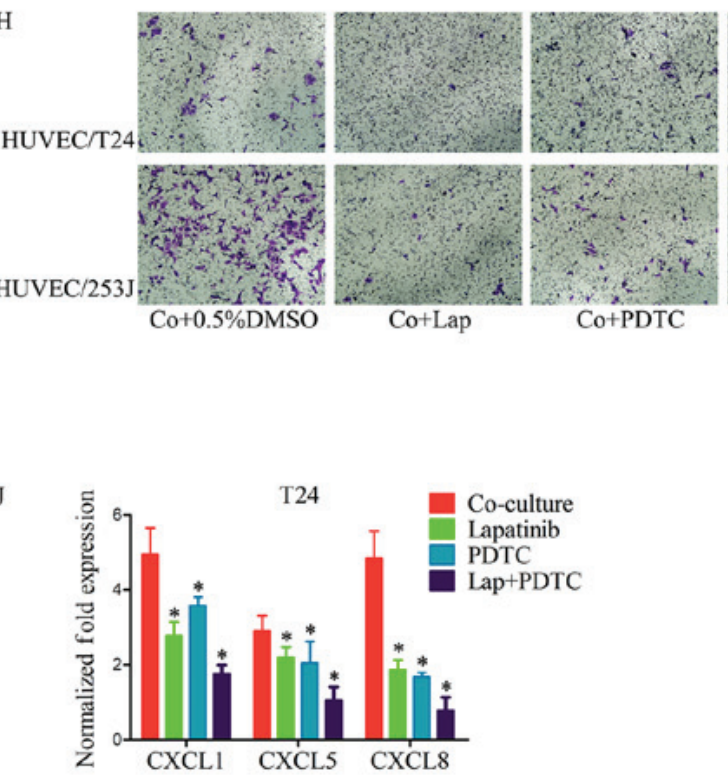

$\mathrm{E}$
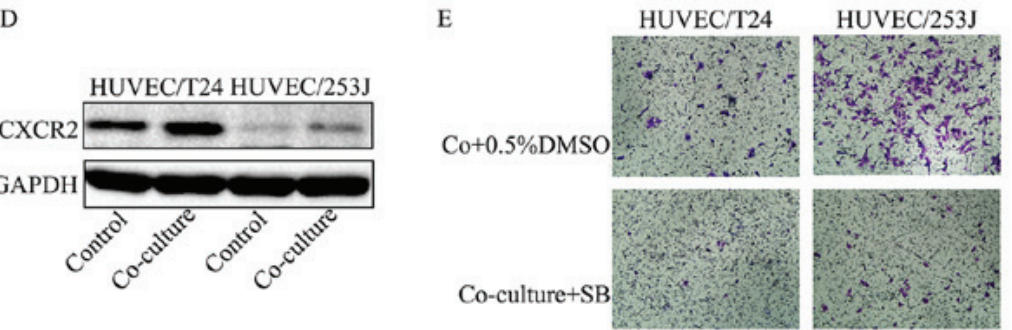

F
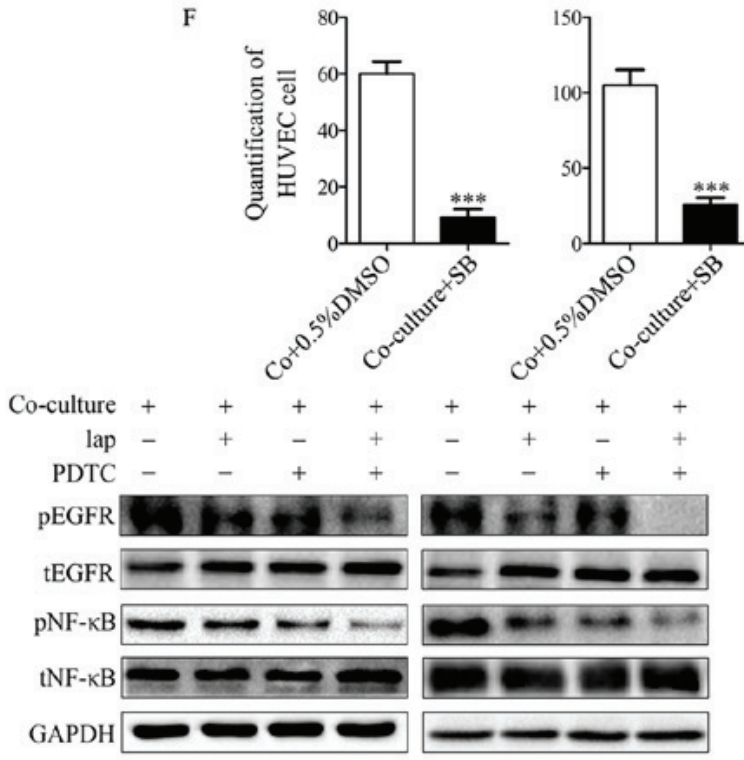

$\begin{array}{ll}+ & + \\ - & +\end{array}$

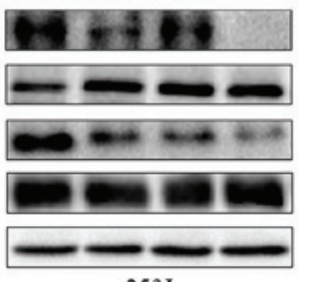

$253 \mathrm{~J}$
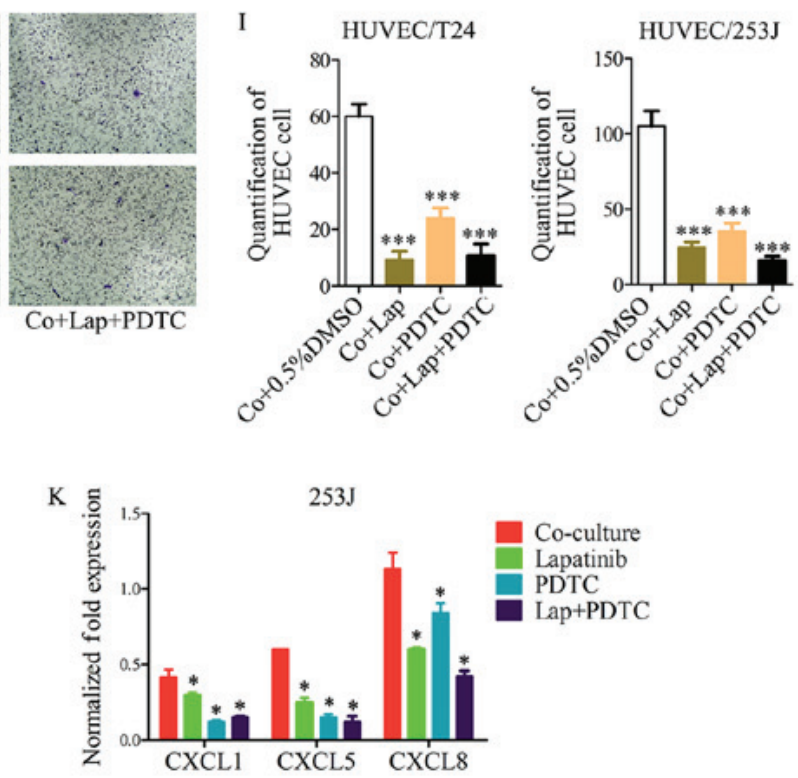

Figure 5. Cancer cell treatment by the co-culture system led to enhanced HUVEC recruitment. (A and B) T24/253J treatment by co-culture with HUVECs and enhanced HUVEC recruitment (magnification, $\mathrm{x} 200$ ). ${ }^{* * * *} \mathrm{P}<0.001$ vs. control. (C) RT-qPCR for screening the expression of CXCL1, CXCL5 and CXCL8 in T24/253J following co-culture with HUVECs. "P<0.05 vs. T24; ${ }^{*} \mathrm{P}<0.05$ vs. 253J. (D) Western blot analysis indicates that the co-culture of HUVECs leads to the upregulation of CXCR2. (E and F) Inhibition of the CXCR2 pathway following co-culture treatment in HUVECs, and reduced HUVEC recruitment (magnification, $\mathrm{x} 200)$. ${ }^{* * *} \mathrm{P}<0.001$ vs. Co+0.5\%DMSO. (G) Western blot analysis indicates inhibition of the EGFR-NF- $\kappa \mathrm{B}$ pathway in T24/253J following co-culture with HUVECs. (H and I) Inhibition of the EGFR-NF- $\kappa$ B pathway in T24/253J following co-culture with HUVECs, and reduced endothelial cell recruitment (magnification, $\mathrm{x} 200)$. ${ }^{* * *} \mathrm{P}<0.001$ vs. Co+0.5\%DMSO. ( $\mathrm{J}$ and K) RT-qPCR for screening the expression of CXCL1, CXCL5 and CXCL8 in T24/253J following co-culture with HUVECs, followed by inhibition of the EGFR-NF- $\kappa \mathrm{B}$ pathway. ${ }^{*} \mathrm{P}<0.05$ vs. co-culture. HUVEC, human umbilical vein endothelial cell; co+0.5\%DMSO, co-culture + $0.5 \%$ dimethyl sulfoxide; RT-qPCR, reverse transcription-quantitative polymerase chain reaction; EGFR, epidermal growth factor receptor; NF, nuclear factor; SB, SB225002; lap, lapatinib; p, phosphorylated; t, total.

co-culture in cancer cells. This previous study also revealed that B cell lymphoma-2 upregulated CXC chemokine expression in ECs through the NF- $\mathrm{KB}$ pathway (24); it is known that the
NF- $\kappa B$ pathway has a key role in the transcription of several cytokines and growth factors (25). These observations led to the hypothesis that in co-culture system, EGFR signaling induces 


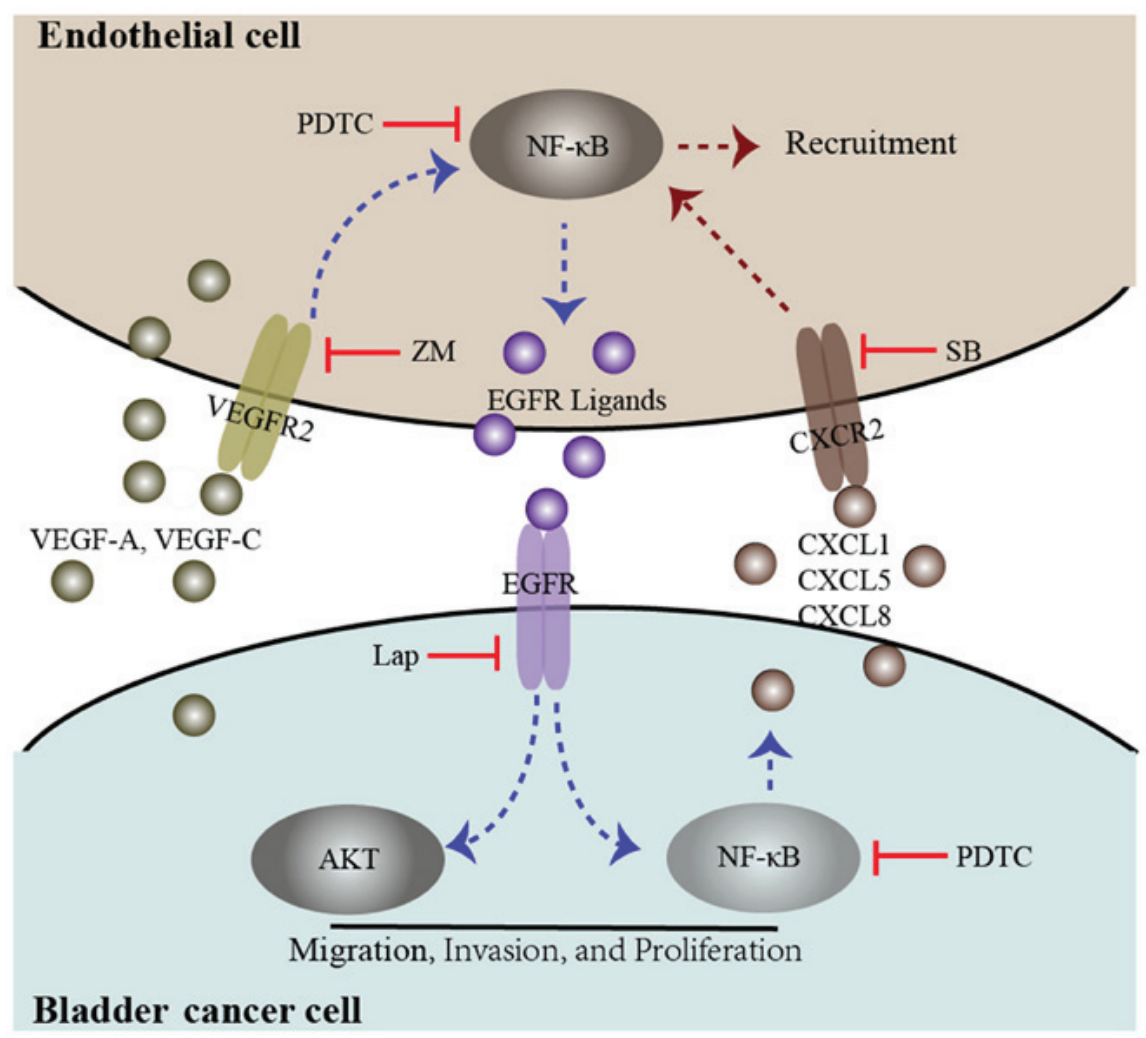

Figure 6. Diagram proposing a model for the interaction between bladder cancer cells and endothelial cells. In the co-culture system, both bladder cancer cells and endothelial cells secrete the VEGFR2 ligands VEGF-A and VEGF-C, which induce VEGFR2 signaling and downstream NF- $\mathrm{kB}$ signaling, promoting EGFR ligand expression. These events may be inhibited by a VEGFR2 inhibitor, ZM and an NF- $\mathrm{kB}$ inhibitor (PDTC). EGFR signaling in bladder cancer cells was triggered by EGFR ligands secreted by endothelial cells, which induces phosphorylation of AKT and NF-кB. These events enhance bladder cancer migration, invasion, and proliferation. Furthermore, activated EGFR signaling in bladder cancer cells could enhance endothelial cell recruitment through the upregulation of CXCL1, CXCL5 and CXCL8. These events could be inhibited by an EGFR inhibitor, lap, PDTC and a CXCR2 inhibitor, SB. VEGF, vascular endothelial growth factor; R, receptor; NF, nuclear factor; EGFR, epidermal growth factor receptor; ZM, ZM 323881 HCL; AKT, protein kinase B; lap, lapatinib; SB, SB225002.

the expression of CXCL1/5/8 in cancer cells and enhances EC recruitment ability. To test this hypothesis, the EGFR signaling and downstream NF- $\mathrm{BB}$ pathway were inhibited; the results demonstrated that the co-culture induced enhanced HUVEC recruitment of T24/253J cells was attenuated in the absence of the EGFR/NF- $\kappa \mathrm{B}$ signaling pathway (Fig. 5G-I). In addition, inhibition of the EGFR/NF- $\mathrm{B}$ signaling pathway in bladder cancer cells though the co-culture system downregulated the gene expression of CXCL1/5/8 (Fig. 5J and K).

\section{Discussion}

The present data suggest a novel site for vascular EC and cancer cell interaction in the tumor microenvironment. It was demonstrated that EC has an active role in promoting cancer cell proliferation, survival, migration and invasion. A number of previous reports suggested a role for macrophages $(26,27)$ and neutrophils (28) in the progression of bladder cancer. However, the role of ECs in the tumor microenvironment in bladder cancer is only beginning to be unveiled. The present study provides evidence for ECs as key players in bladder cancer progression.

The present results suggest that ECs have an active role in bladder cancer. It was demonstrated that EGFR was phosphorylated in bladder cancer cells by the EC-secreted EGFR ligands in the co-culture system. This finding is in accordance with observations from other reports that have characterized growth factors as critical in cancer progression $(29,30)$. These findings demonstrated that EGFR ligands, including epidermal growth factor (EGF), amphiregulin, epiregulin, betacellulin, tumor necrosis factor- $\alpha$ and epithelial mitogen are upregulated in EC by co-culturing $(28,29)$. EGFR overexpression has been identified in a variety of tumors, including non-small cell lung cancer (31), breast cancer (32), prostate cancer (33) and bladder cancer (34). The expression of EGFR ligands and EGFR is correlated with cancer proliferation, survival, and metastasis. For example, cancer cell proliferation, migration, and invasion are increased through the EGFR-Ras/Raf/MEK/ERK and EGFR-PI3K/AKT pathways (35). A previous study has demonstrated that vascular ECs secrete CXCL8, IL-6 and EGF following co-culture with head and neck squamous cell carcinoma cells. It was observed that the primary effect of EC-derived IL-6 was the activation of STAT3, the primary effect of CXCL8 was on the activity of AKT, and the primary effect of EGF was on ERK activity in head and neck squamous cell carcinoma cells (15). However, the present results indicated that $\mathrm{AKT}$ and $\mathrm{NF}-\kappa \mathrm{B}$ pathways were downstream of EGFR signaling, and can be activated by EGFR signaling in the co-culture system.

In the tumor vasculature, VEFGR2 expression is upregulated compared with normal vasculature (22). In addition, VEGFR2 expression is a prognostic marker for the 
clinical outcome of patients (36). It has been demonstrated previously that stimulation with VEGF upregulated a number of genes in vascular EC. For example, Ets-1, MMP1 and Fil-1 were stimulated by VEGF via VEGFR2 signaling (37). In the present study, it was demonstrated that VEGFR2 signaling was activated by co-culture, as was downstream $\mathrm{NF}-\kappa \mathrm{B}$. VEGFR2 was inhibited in the vascular ECs following co-culture education, and the results indicated that the expression of EGFR ligands is regulated through the VEGFR2/NF- $\kappa$ B pathway in the co-culture system. The findings also demonstrated that the expression of both VEGF-A and VEGF-C in cancer cells and vascular ECs is upregulated following co-culture treatment. The mechanism behind this remains to be elucidated. The ELR ${ }^{+}$chemokine has been reported to induce vascular EC migration in vitro and promote angiogenesis in vivo (38). A previous study revealing that the $\mathrm{ELR}^{+}$chemokines CXCL5 and CXCL8 bind to $\mathrm{CXCR} 2$ and induce neovascularization, and that the neutralization of CXCL5 or CXCL8 using specific neutralizing antibodies against these small molecules may inhibit the chemokine mediated angiogenesis (39).

In the present study, the expression levels of CXCL1, CXCL5, and CXCL8 were upregulated in bladder cancer cells by co-culture treatment, and the expression of CXCR 2 was upregulated in ECs. Following co-culture treatment CXCR2 was inhibited using SB225002. These results indicated that vascular EC interactions with bladder cancer cells induce vascular EC recruitment though the CXCL1/CXCL5/CXCL8-CXCR2 pathway. Multiple signaling pathways contribute to CXCL1, CXCL5, and CXCL8 regulation. EGFR ligands, for example transforming growth factor- $\alpha$ and amphiregulin, induce CXCL8 expression in bronchial epithelial cells and mediate cigarette smoke-induced CXCL8 expression through an autocrine loop $(40,41)$. CXCL1 is critical in cancer progression and a previous study revealed that EGF activation of the PI3K pathway induced the expression of CXCL1 (42). Furthermore, the expression of CXCL5 has been reported to be upregulated by $\mathrm{NF}-\kappa \mathrm{B}$ family members, or by the $\mathrm{p} 53$ pathway $(25,43)$. In the present study, the activation of EGFR signaling in the co-culture system was inhibited using lapatinib, and the results indicated that EGFR ligands were involved in the regulation of CXCL1, CXCL5 and CXCL8. In addition, the EGFR pathway and/or the $\mathrm{NF}-\kappa \mathrm{B}$ pathway were inhibited in the co-culture system, and the results indicated that CXCL1, CXCL5 and CXCL8 were upregulated in bladder cancer cells through the EGFR pathway. A summary of the present study is presented in Fig. 6.

In conclusion, the present study demonstrated that interactions of bladder cancer cells with vascular ECs enhance vascular EC recruitment by cancer cells through the CXC chemokine and CXCR2 signaling pathways. The recruited vascular ECs interact with bladder cancer cells and tissues to promote cancer progression through the EGFR signaling pathway. The present study may also illuminate mechanisms by which the tumor microenvironment promotes malignant progression in other cancer types, including colon, prostate and breast cancer.

\section{Acknowledgements}

Not applicable.

\section{Funding}

This study was supported by grants from the National Natural Science Foundation of China (grant no. 81572520) and the Key Science and Technology Program of Shaanxi Province, China (grant no. 2015SF176).

\section{Availability of data and materials}

The analyzed datasets generated during the present study are available from the corresponding author on reasonable request.

\section{Authors' contributions}

$\mathrm{ZH}$ wrote the main manuscript. $\mathrm{ZH}, \mathrm{MZ}, \mathrm{GC}$ and $\mathrm{YY}$ performed the experiments. JF, ZG and $\mathrm{ZH}$ designed the study. WW, XW and PZ performed data analysis. ZH and JF contributed to manuscript revisions. All authors reviewed the manuscript. All authors read and approved the final manuscript

\section{Ethics approval and consent to participate}

Not applicable.

\section{Patient consent for publication}

Not applicable.

\section{Competing interests}

The authors declare that they have no conflicts of interest.

\section{References}

1. Chavan S, Bray F, Lortet-Tieulent J, Goodman M and Jemal A: International variations in bladder cancer incidence and mortality. Eur Urol 66: 59-73, 2014.

2. Smith ZL and Guzzo TJ: Urinary markers for bladder cancer. F1000Prime Rep 5: 21, 2013.

3. Calderaro J, Rebouissou S, de Koning L, Masmoudi A, Hérault A, Dubois T, Maille P, Soyeux P, Sibony M, de la Taille A, et al: PI3K/AKT pathway activation in bladder carcinogenesis. Int $\mathrm{J}$ Cancer 134: 1776-1784, 2014.

4. Ching CB and Hansel DE: Expanding therapeutic targets in bladder cancer: The PI3K/Akt/mTOR pathway. Lab Invest 90: 1406-1414, 2010.

5. López-Knowles E, Hernández S, Malats N, Kogevinas M, Lloreta J, Carrato A, Tardón A, Serra C and Real FX: PIK3CA mutations are an early genetic alteration associated with FGFR3 mutations in superficial papillary bladder tumors. Cancer Res 66: 7401-7404, 2006

6. Wu X, Obata T, Khan Q, Highshaw RA, De Vere White R and Sweeney C: The phosphatidylinositol-3 kinase pathway regulates bladder cancer cell invasion. BJU Int 93: 143-150, 2004.

7. Oka N, Tanimoto S, Taue R, Nakatsuji H, Kishimoto T, Izaki H, Fukumori T, Takahashi M, Nishitani M and Kanayama HO: Role of phosphatidylinositol-3 kinase/Akt pathway in bladder cancer cell apoptosis induced by tumor necrosis factor-related apoptosis-inducing ligand. Cancer Sci 97: 1093-1098, 2006.

8. Mukherjee N, Houston TJ, Cardenas E and Ghosh R: To be an ally or an adversary in bladder cancer: The NF- $\kappa B$ story has not unfolded. Carcinogenesis 36: 299-306, 2015.

9. Guan Z, Ding C, Du Y, Zhang K, Zhu JN, Zhang T, He D, Xu S, Wang $X$ and Fan J: HAF drives the switch of HIF-1 $\alpha$ to HIF-2 $\alpha$ by activating the $\mathrm{NF}-\kappa \mathrm{B}$ pathway, leading to malignant behavior of T24 bladder cancer cells. Int J Oncol 44: 393-402, 2014.

10. Bennasroune A, Gardin A, Aunis D, Crémel G and Hubert P: Tyrosine kinase receptors as attractive targets of cancer therapy. Crit Rev Oncol Hematol 50: 23-38, 2004. 
11. Folkman J: Role of angiogenesis in tumor growth and metastasis. Semin Oncol 29 (Suppl 16): 15-18, 2002.

12. Sparmann A and Bar-Sagi D: Ras-induced interleukin- 8 expression plays a critical role in tumor growth and angiogenesis. Cancer Cell 6: 447-458, 2004.

13. Warner KA, Miyazawa M, Cordeiro MMR, Love WJ, Pinsky MS, Neiva KG, Spalding AC and Nör JE: Endothelial cells enhance tumor cell invasion through a crosstalk mediated by CXC chemokine signaling. Neoplasia 10: 131-139, 2008.

14. Zeng Q, Li S, Chepeha DB, Giordano TJ, Li J, Zhang H, Polverini PJ, Nor J, Kitajewski J and Wang CY: Crosstalk between tumor and endothelial cells promotes tumor angiogenesis by MAPK activation of Notch signaling. Cancer Cell 8: 13-23, 2005.

15. Zhang Z, Dong Z, Lauxen IS, Filho MS and Nör JE: Endothelial cell-secreted EGF induces epithelial to mesenchymal transition and endows head and neck cancer cells with stem-like phenotype. Cancer Res 74: 2869-2881, 2014.

16. Ferrara N, Hillan KJ, Gerber HP and Novotny W: Discovery and development of bevacizumab, an anti-VEGF antibody for treating cancer. Nat Rev Drug Discov 3: 391-400, 2004

17. Shaulian E and Karin M: AP-1 as a regulator of cell life and death. Nat Cell Biol 4: E131-E136, 2002.

18. Livak KJ and Schmittgen TD: Analysis of relative gene expression data using real-time quantitative PCR and the 2(-Delta Delta C(T)) method. Methods 25: 402-408, 2001

19. Fus LP and Górnicka B: Role of angiogenesis in urothelial bladder carcinoma. Cent European J Urol 69: 258-263, 2016.

20. Rajjayabun PH,Keegan PE, Lunec J and Mellon JK: erbB receptor expression patterns in human bladder cancer. Urology 66 196-200, 2005

21. Nicholson RI, Gee JM and Harper ME: EGFR and cancer prognosis. Eur J Cancer 37 (Suppl 4): S9-S15, 2001.

22. Plate KH, Breier G, Millauer B, Ullrich A and Risau W: Up-regulation of vascular endothelial growth factor and its cognate receptors in a rat glioma model of tumor angiogenesis. Cancer Res 53: 5822-5827, 1993.

23. Holmes K, Roberts OL, Thomas AM and Cross MJ: Vascular endothelial growth factor receptor-2: Structure, function, intracellular signalling and therapeutic inhibition. Cell Signal 19: 2003-2012, 2007.

24. Kaneko T, Zhang Z, Mantellini MG, Karl E, Zeitlin B, Verhaegen M, Soengas MS, Lingen M, Strieter RM, Nunez G, et al: Bcl-2 orchestrates a cross-talk between endothelial and tumor cells that promotes tumor growth. Cancer Res 67: 9685-9693, 2007

25. Richmond A: Nf-kappa B, chemokine gene transcription and tumour growth. Nat Rev Immunol 2: 664-674, 2002

26. Miyake M, Hori S, Morizawa Y, Tatsumi Y, Nakai Y, Anai S, TorimotoK,Aoki K, TanakaN,Shimada K, et al:CXCL1-mediated interaction of cancer cells with tumor-associated macrophages and cancer-associated fibroblasts promotes tumor progression in human bladder cancer. Neoplasia 18: 636-646, 2016.

27. Tian YF, Tang K, Guan W, Yang T, Xu H, Zhuang QY and Ye ZQ: OK-432 suppresses proliferation and metastasis by tumor associated macrophages in bladder cancer. Asian Pac J Cancer Prev 16: 4537-4542, 2015.

28. Lin C, Lin W, Yeh S, Li L and Chang C: Infiltrating neutrophils increase bladder cancer cell invasion via modulation of androgen receptor (AR)/MMP13 signals. Oncotarget 6: 43081-43089, 2015

29. Gunes S, Sullu Y, Yegin Z, Buyukalpelli R, Tomak L and Bagci H: ErbB receptor tyrosine kinase family expression levels in urothelial bladder carcinoma. Pathol Res Pract 209: 99-104, 2013.
30. Appert-Collin A, Hubert P, Crémel G and Bennasroune A: Role of ErbB receptors in cancer cell migration and invasion. Front Pharmacol 6: 283, 2015

31. Gately K, Forde L, Cuffe S, Cummins R, Kay EW, Feuerhake F and O'Byrne KJ: High coexpression of both EGFR and IGF1R correlates with poor patient prognosis in resected non-small-cell lung cancer. Clin Lung Cancer 15: 58-66, 2014.

32. Lee HJ, Seo AN, Kim EJ, Jang MH, Kim YJ, Kim JH, Kim SW, Ryu HS, Park IA, Im SA, et al: Prognostic and predictive values of EGFR overexpression and EGFR copy number alteration in HER2-positive breast cancer. Br J Cancer 112: 103-111, 2015.

33. Day KC, Lorenzatti Hiles G, Kozminsky M, Dawsey SJ, Paul A, Broses LJ, Shah R, Kunja LP, Hall C, Palanisamy N, et al: HER2 and EGFR overexpression support metastatic progression of prostate cancer to bone. Cancer Res 77: 74-85, 2017.

34. Lipponen $\mathrm{P}$ and Eskelinen $\mathrm{M}$ : Expression of epidermal growth factor receptor in bladder cancer as related to established prognostic factors, oncoprotein (c-erbB-2, p53) expression and long-term prognosis. Br J Cancer 69: 1120-1125, 1994.

35. Lo HW and Hung MC: Nuclear EGFR signalling network in cancers: Linking EGFR pathway to cell cycle progression, nitric oxide pathway and patient survival. Br J Cancer 94: 184-188, 2006.

36. Fine BA, Valente PT, Feinstein GI and Dey T: VEGF, flt-1, and $\mathrm{KDR} / \mathrm{flk}-1$ as prognostic indicators in endometrial carcinoma. Gynecol Oncol 76: 33-39, 2000.

37. Sato Y, Kanno S, Oda N, Abe M, Ito M, Shitara K and Shibuya M: Properties of two VEGF receptors, Flt-1 and KDR, in signal transduction. Ann NY Acad Sci 902: 201-205, discussion 205-207, 2000.

38. Strieter RM, Polverini PJ, Kunkel SL, Arenberg DA, Burdick MD, Kasper J, Dzuiba J, Van Damme J, Walz A, Marriott D, et al: The functional role of the ELR motif in CXC chemokine-mediated angiogenesis. J Biol Chem 270: 27348-27357, 1995.

39. Addison CL, Daniel TO, Burdick MD, Liu H, Ehlert JE, Xue YY Buechi L, Walz A, Richmond A and Strieter RM: The CXC chemokine receptor 2, CXCR2, is the putative receptor for $\mathrm{ELR}^{+}$ CXC chemokine-induced angiogenic activity. J Immunol 165: 5269-5277, 2000

40. Subauste MC and Proud D: Effects of tumor necrosis factor-alpha, epidermal growth factor and transforming growth factor-alpha on interleukin-8 production by, and human rhinovirus replication in bronchial epithelial cells. Int Immunopharmacol 1: 1229-1234, 2001.

41. Richter A, O'Donnell RA, Powell RM, Sanders MW, Holgate ST, Djukanović R and Davies DE: Autocrine ligands for the epidermal growth factor receptor mediate interleukin-8 release from bronchial epithelial cells in response to cigarette smoke. Am J Respir Cell Mol Biol 27: 85-90, 2002.

42. Moscova M, Marsh DJ and Baxter RC: Protein chip discovery of secreted proteins regulated by the phosphatidylinositol 3-kinase pathway in ovarian cancer cell lines. Cancer Res 66: 1376-1383, 2006.

43. Yeudall WA, Vaughan CA, Miyazaki H, Ramamoorthy M, Choi MY, Chapman CG, Wang H, Black E, Bulysheva AA, Deb SP, et al: Gain-of-function mutant p53 upregulates CXC chemokines and enhances cell migration. Carcinogenesis 33: 442-451, 2012.

This work is licensed under a Creative Commons Attribution-NonCommercial-NoDerivatives 4.0 International (CC BY-NC-ND 4.0) License. 